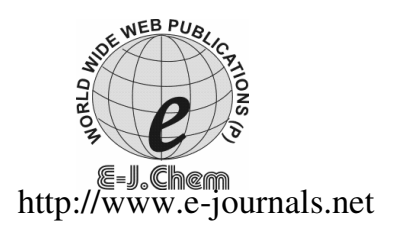

ISSN: 0973-4945; CODEN ECJHAO

E-Journal of Chemistry

2010, 7(4), 1212-1219

\title{
The Preparation and Characterization of Chitosan / Poly (Vinyl Alcohol) Blended Films
}

\author{
ESAM A. EL-HEFIAN*, MOHAMED MAHMOUD NASEF ${ }^{\S}$ and \\ ABDUL HAMID YAHAYA \\ Department of Chemistry, Faculty of Science, \\ University of Malaya, 50603 Kuala Lumpur, Malaysia. \\ ${ }^{\S}$ Chemical Engineering Department, Faculty of Chemical and Natural Resources \\ Engineering, Universiti Teknologi Malaysia, 81310 UTM Skudai, Johor, Malaysia. \\ eelhefian@yahoo.com
}

Received 5 December 2009; Accepted 2 February 2010

\begin{abstract}
In this study, chitosan and PVA were blended at different proportions (considering chitosan as the main component) in solution forms. The chemical structure and the morphology of the obtained blend films were investigated using FTIR and field emission scanning electron microscope (FESEM). The thermal stability of the blend films were also studied using thermal gravimetric analysis (TGA). Our results showed that chitosan and PVA form a compatible blend and their films displayed homogenous and smooth surface properties compared to their individual pure components. The blending of PVA with chitosan at all proportions was found to highly enhance the swelling of the obtained films compared to that of pure chitosan one.
\end{abstract}

Keywords: Chitosan, Poly (Vinyl alcohol), Blending, Films.

\section{Introduction}

Chitosan is a natural polysaccharide formed by alkaline deacetylation of the second most abundant naturally occurring chitin of crab and shrimp shells ${ }^{1}$. Due to the amino groups that chitosan possesses in its chain, it can be dissolved in dilute aqueous acid solutions, such as acetic acid and propionic acid. Since it is inexpensive, non-toxic and possesses potentially reactive amino functional groups, chitosan has been evaluated for numerous applications, including medicine, food, cosmetics and wastewater treatment ${ }^{2-6}$.

Poly (vinyl alcohol) is a non-toxic, water-soluble synthetic polymer that has good filmforming ability. It has a large number of hydroxyl groups which allows it to react with many types of functional groups. This advantage makes it suitable for biocompatible materials. PVA has been widely used on biomaterial applications ${ }^{7}$. 
To improve chitosan properties and further diversify its applications, various strategies have been adopted. This includes: (1) crosslinking ${ }^{8}$ (2) graft copolymerization, ${ }^{9,10}$, (3) complexation $^{11}$, (4) chemical modifications ${ }^{12}$ and (5) blending ${ }^{13,14}$. In particular, the modification of chitosan by means of blending is an attractive method that has been extensively used for providing new desirable characters to chitosan ${ }^{15,16}$. This is mainly due to its simplicity, availability of wide range of synthetic and natural polymers for blending and effectiveness for practical utilization.

In this work, we report on the preparation of chitosan/poly (vinyl alcohol) films obtained by the physical solution blending of chitosan and PVA at different proportions. FTIR spectral analysis was used to confirm the presence of functional groups of both chitosan and PVA in the blend. The properties of various blended films of CS/PVA, such as swelling, thermal stability and morphology were also investigated.

\section{Experimental}

Chitosan with a degree of deacetylation (DD) of $88.1 \%$ determined by UV method ${ }^{17}$ was purchased from a commercial source. PVA Fluka (56-98) with an average molecular weight of $195 \times 10^{3} \mathrm{~g} \mathrm{~mol}^{-1}$ was also used in this work. Acetic acid (glacial 100\%, pro-analysis) was purchased from Merck (Darmstadt, Germany). Ultra pure water (Maxima Ultra Pure Water, Elga-Prima Corp, UK) with a resistivity greater than $18 \mathrm{M} \Omega / \mathrm{cm}$ was used to prepare all solutions. All chemicals were used without further purification and freshly prepared solutions were used in all experiments.

\section{Preparation of the solutions}

The chitosan was dried in an oven until a constant weight was observed. A $10 \mathrm{~g} \mathrm{~L}^{-1}$ solution of chitosan was prepared by dissolving $5 \mathrm{~g}$ of chitosan in $500 \mathrm{~mL}$ acetic acid $(0.1 \mathrm{M})$ followed by stirring and heating at $60{ }^{\circ} \mathrm{C}$ overnight. The solution was filtered to remove dust and other traces of impurities. Air bubbles were eliminated by keeping the solutions at room temperature for $2 \mathrm{~h}$.

A similar $10 \mathrm{~g} \mathrm{~L}^{-1}$ solution of PVA was prepared by dissolving $5 \mathrm{~g}$ of PVA in $500 \mathrm{~mL}$ of preheated ultra pure water. The solution was then stirred and kept at about $90{ }^{\circ} \mathrm{C}$ for $2 \mathrm{~h}$.

\section{Preparation of the blended films}

The preparation of blended films of chitosan and PVA was carried out at various proportions. The aqueous PVA solution was added drop by drop to the chitosan solution, under continuous stirring at around $94{ }^{\circ} \mathrm{C}$. Stirring was allowed to continue for $30 \mathrm{~min}$ after mixing and the range of the added PVA to chitosan solution was from 0-50 vol\%. Films of the resulting homogeneous solutions were obtained by casting prescribed amounts of the solutions onto polystyrene petri dishes followed by drying at $60{ }^{\circ} \mathrm{C}$ for $48 \mathrm{~h}$. The films were peeled off and kept under evacuated desiccator over fresh silica gel until use. All films obtained were transparent and free of air bubbles.

Similar films from pure chitosan and pure PVA were prepared using the same casting procedure and used as references. The chitosan films were neutralized with $0.1 \mathrm{M} \mathrm{NaOH}$ solution overnight washed thoroughly with distilled water and dried subsequently.

\section{Film thickness}

The film thickness was measured with a digital micrometer (Mitutoyo, Japan) with $0.001 \mathrm{~mm}$ resolution. Several thickness measurements were taken at several points of the film and then, the average was calculated. 


\section{Instrumentation}

\section{Molecular weight measurements}

The molecular weight of chitosan was about $5.5 \times 10^{5} \mathrm{~g} \mathrm{~mol}^{-1}$ determined at $30^{\circ} \mathrm{C}$ by gel permeation chromatography (GPC) equipped with a Waters 1515 HPLC Pump and a Waters 2414 Refractive Index Detector. The column used was PL aquagel-OH 30 $(8 \mu \mathrm{m}, 300 \times 7.5 \mathrm{~mm})$ and the solvent used was $1 \%$ acetic acid. The GPC was calibrated using pullulans as standard.

\section{FTIR measurements}

FTIR measurements of blended films (about $10 \mu \mathrm{m}$ thick) were performed on a spectrometer (Perkin Elmer, model 2000). The spectra were obtained at a frequency range of $4000-400 \mathrm{~cm}^{-1}$ with a resolution of $4 \mathrm{~cm}^{-1}$ and 8-times scanning.

\section{FESEM measurements}

The surface morphology was investigated by a FEI Quanta 200F field emission scanning electron microscope (FESEM) (FEI, USA) controlled by a 32 bit computer system using Microsoft Windows 2000 as an operating system.

\section{Thermogravimetric measurements}

TGA was used to evaluate the thermal stability and to determine the decomposition temperature of chitosan. Thermogravimetric measurements were performed using a MettlerToledo thermogravimetric analyzer model TGA/SDTA851e. TGA runs were carried out in a temperature range of $30-500{ }^{\circ} \mathrm{C}$, under nitrogen atmosphere and with a constant heating rate of $10^{\circ} \mathrm{C} / \mathrm{min}$ in all the experiments. The sample size of $4-10 \mathrm{mg}$ was weighed and the mass of the sample pan was continuously recorded as a function of temperature.

\section{Degree of swelling}

The swelling behavior of the films was measured by immersing the blended films in distilled water at room temperature for $10 \mathrm{~h}$. The excess water on the surface of the films was removed by blotting the surface with tissue paper and the weight was then recorded. This process was repeated at least three times for each sample. The degree of swelling was calculated using the following equation:

$$
\text { Degree of swelling }(\%)=\left[\left(\mathrm{W}_{2}-\mathrm{W}_{1}\right) / \mathrm{W}_{1}\right] \times 100
$$

where, $\mathrm{W}_{1}$ is the weight of completely dried sample and $\mathrm{W}_{2}$ is the weight of swelled sample.

\section{Results and Discussion}

\section{FTIR analysis of pure CS and pure PVA films}

As shown in Figure 1, the spectrum of pure chitosan film shows a broad band at $3367 \mathrm{~cm}^{-1}$ which is due to the $\mathrm{OH}$ stretching. The band at $1560 \mathrm{~cm}^{-1}$ is assigned for the $\mathrm{NH}$ bending (amide II) $\left(\mathrm{NH}_{2}\right)$ while the small peak at $1647 \mathrm{~cm}^{-1}$ is attributed to the $\mathrm{C}=\mathrm{O}$ stretching (amide I) $\mathrm{O}=\mathrm{C}-\mathrm{NHR}$. The bands at 2927, 2884, 1411, 1321 and $1260 \mathrm{~cm}^{-1}$ are assigned to $\mathrm{CH}_{2}$ bending due to pyranose ring ${ }^{18}$. The band at $1380 \mathrm{~cm}^{-1}$ is due to $\mathrm{CH}_{3}$ wagging. The characteristic features of chitosan spectrum in this study are similar to that of previous reports ${ }^{19-21}$. Figure 1 also presents the FTIR spectrum of the pure PVA film. The spectrum shows an absorption peak at $3368 \mathrm{~cm}^{-1}$ which refers to the intermolecular hydrogen bonding and $-\mathrm{OH}$ stretch vibration ${ }^{22}$. The vibrational band observed at $2941 \mathrm{~cm}^{-1}$ is associated with the $\mathrm{C}-\mathrm{H}$ stretching from alkyl groups. The absorption corresponding to the $-\mathrm{C}-\mathrm{O}$ stretching occurs at $1096 \mathrm{~cm}^{-1}$. 


\section{FTIR Analysis of CS/PVA blended films}

Figure 1 shows the FTIR spectra of chitosan and PVA blended films containing various PVA proportions. A summary of characteristic bands of chitosan and PVA blended films is also presented in Table 1. As can be seen, the increase in the PVA concentration in the blended films caused a decrease in the intensity of the band arising from the NH bending (amide II) at $1561 \mathrm{~cm}^{-1}$ of chitosan. Also, an increase in the intensity of $\mathrm{CH}$ group at around $2928 \mathrm{~cm}^{-1}$ was observed as the PVA content increases. In addition, there was a remarkable shift for the peak at $1077 \mathrm{~cm}^{-1}$ to a higher wave number with the increase of PVA content in the blend. Furthermore, the band at $850 \mathrm{~cm}^{-1}$ disappeared in the spectra of the pure chitosan film and the chitosan/PVA blend film containing PVA concentration of $10 \%$.

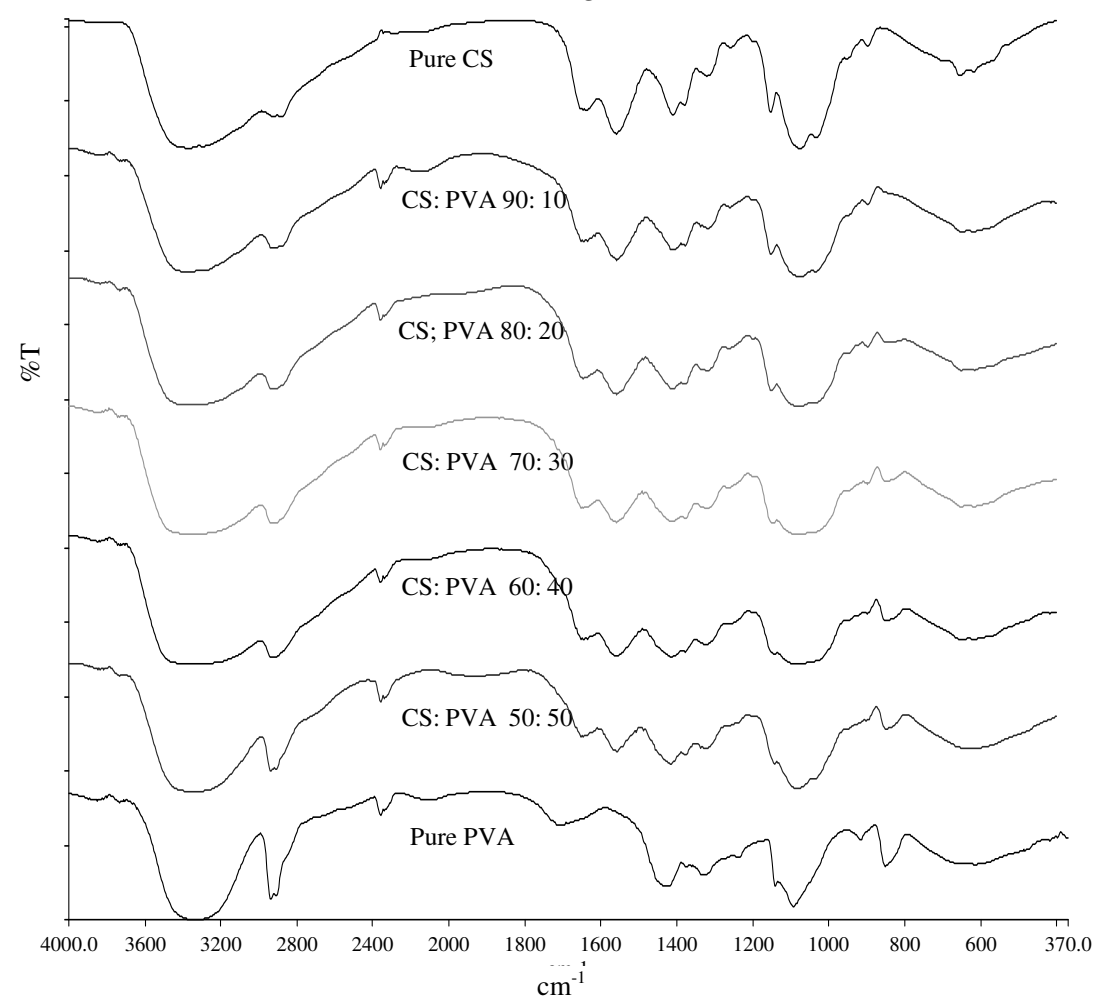

Figure 1. Typical FTIR spectra of pure chitosan, pure PVA and their blended films having various proportions

Table 1. FTIR characteristic bands of chitosan/PVA blend films

\begin{tabular}{ccccccccc}
\hline CS/PVA, \% & C-C & C-O & C-O stretch & CH & NH amide II & C=O amide I & CH & OH \\
\hline $100 / 0$ & - & 1033 & 1077 & 1380 & 1561 & 1648 & 2928 & 3368 \\
$90 / 10$ & - & 1035 & 1077 & 1386 & 1559 & 1647 & 2927 & 3367 \\
$80 / 20$ & 850 & 1035 & 1078 & 1379 & 1559 & 1647 & 2935 & 3367 \\
$70 / 30$ & 851 & 1036 & 1079 & 1379 & 1559 & 1647 & 2937 & 3367 \\
$60 / 40$ & 850 & 1039 & 1082 & 1380 & 1559 & 1647 & 2939 & 3367 \\
$50 / 50$ & 851 & 1037 & 1087 & 1379 & 1559 & 1647 & 2940 & 3367 \\
$0 / 100$ & 852 & - & 1094 & 1375 & - & - & 2941 & 3368 \\
\hline
\end{tabular}


However, the intensity of this band was increasing for the other ratios as the content of PVA increased. This is due to the fact that when two or more polymers are mixed, changes in characteristic spectra peaks occur due to the reflection of the physical blends and chemical interactions ${ }^{23,24}$. These observations indicate the existence of good miscibility between chitosan and PVA and this is most likely due to the formation of intermolecular hydrogen bonds between the amino and hydroxyl groups in chitosan and the hydroxyl groups in PVA.

\section{FESEM measurements of CS/PVA blended films}

Figure 2 shows the SEM micrographs of surfaces of chitosan/PVA blended films containing different proportions of PVA together with the corresponding ones of pure chitosan and PVA films. As can be seen, chitosan film shows smooth and homogeneous surface with some straps unlike that of pure PVA where no straps are seen. The surfaces of the blended films of chitosan and PVA are homogeneous with no pores ${ }^{25}$ and have no interface layer. The blended films also exhibit flat smooth surface in general indicates the uniform distribution of CS and PVA molecules throughout the films. The formation of homogeneous blends of chitosan and PVA was mostly caused by the interactions of hydrogen bonds between the functional groups of the blended component $\left(-\mathrm{OH}\right.$ and $-\mathrm{NH}_{2}$ groups in chitosan and -OH groups in PVA).

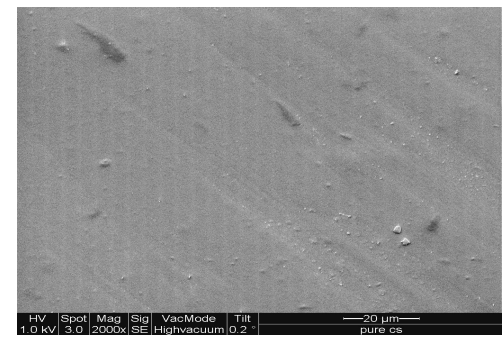

$\mathrm{a}$

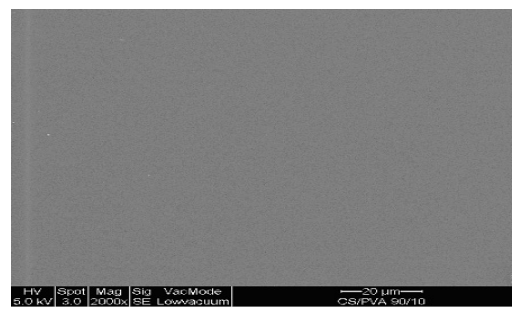

C

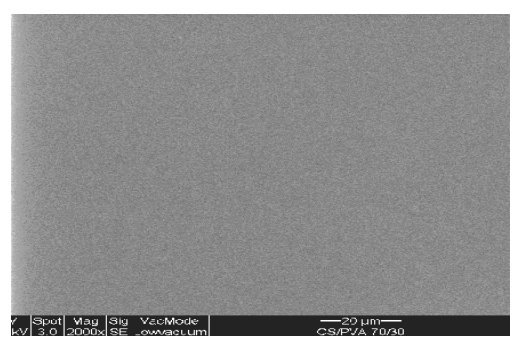

e

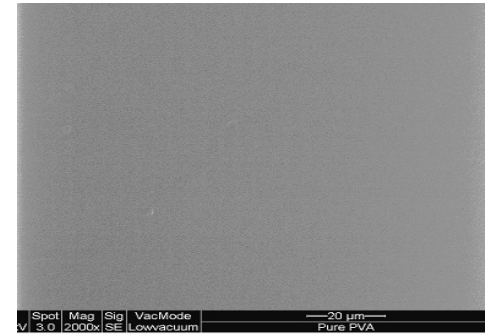

b

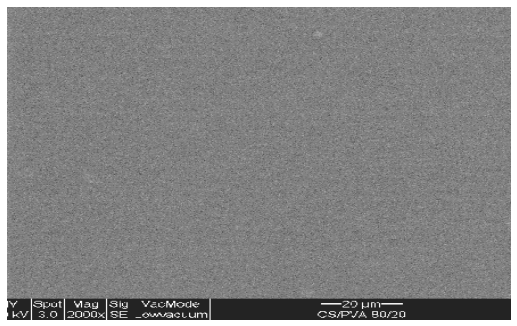

d

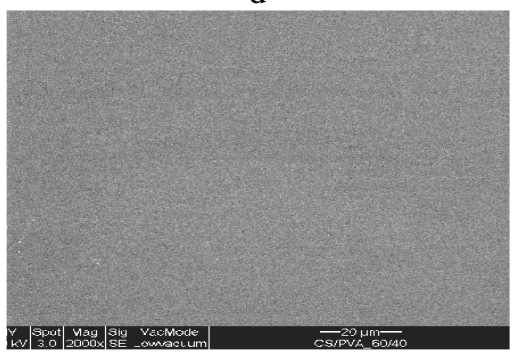

f 


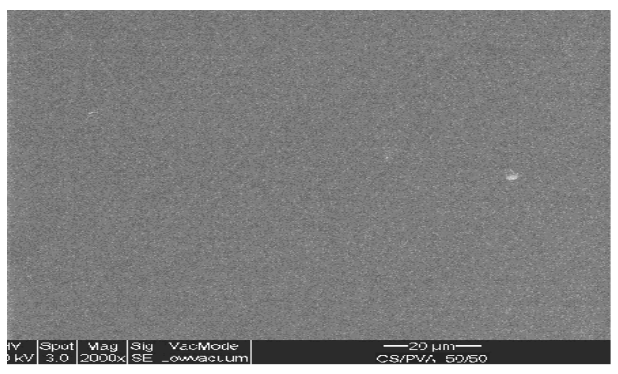

$\mathrm{g}$

Figure 2. The scanning electron micrographs of pure CS film, pure PVA film and CS/PVA blend films; (a) pure CS, (b) pure PVA, (c) 90/10, (d) 80/20, (e) 70/30, (f) 60/40 and (g) 50/50

\section{TGA of pure chitosan film, pure PVA film and their blended films}

The representative TGA curves for the pure CS, pure PVA and CS/PVA blended films are shown in Figure 3. A two-stage weight loss was recorded for the chitosan film. The first transition falls in the range of $40{ }^{\circ} \mathrm{C}-130{ }^{\circ} \mathrm{C}$ and shows about $6 \%$ loss in weight. This is due to the moisture vaporization inside the film. The second weight loss begins at about $150{ }^{\circ} \mathrm{C}$ and the corresponding weight loss of about $52 \%$, is attributed to the decomposition of chitosan. The total weight loss of the sample at about $500{ }^{\circ} \mathrm{C}$ is $58 \%$. Similar behavior of chitosan film was reported in the literature $\mathrm{e}^{26-28}$.

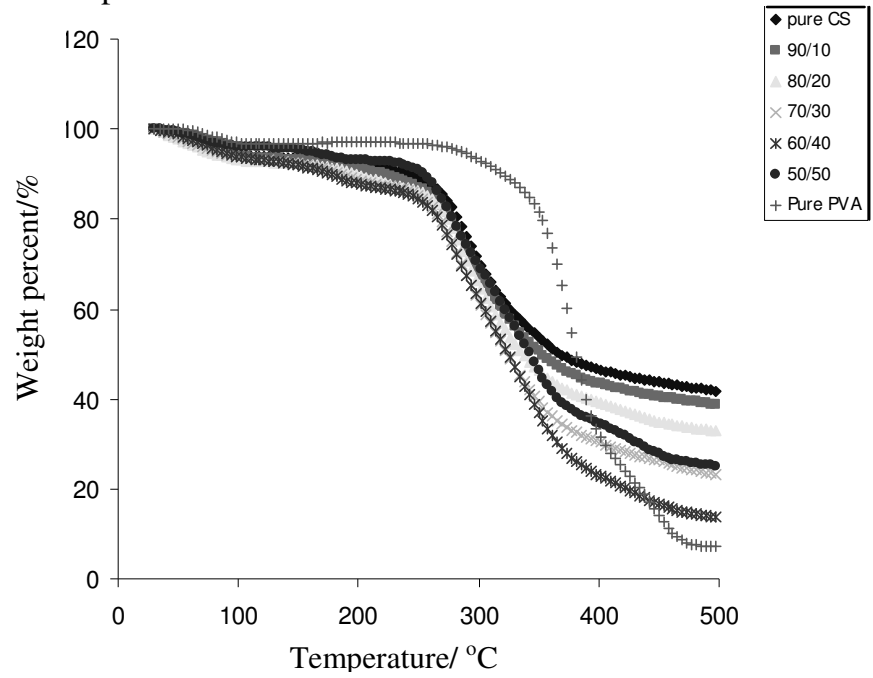

Figure 3. TGA thermograms of pure CS film, pure PVA film and their blended films having various proportions

As it can be seen, the pure PVA film exhibits a three-step degradation pattern. The first step began around $40{ }^{\circ} \mathrm{C}$ up to $79{ }^{\circ} \mathrm{C}$ with a weight loss up to $3 \%$. The second and the major mass loss is between around $260{ }^{\circ} \mathrm{C}$ to $430{ }^{\circ} \mathrm{C}$ with a weight loss of about $73 \%$. This was followed by a further smaller mass loss (around 17\%) in the third step (from about $430{ }^{\circ} \mathrm{C}$ to $480{ }^{\circ} \mathrm{C}$ ). The first step of weight loss could be attributed to the evaporation of loosely bound water. The second step is predominantly caused by the heat decomposition of the polymer structure. With further heating, the polymer backbone is broken down. Similar observations have been reported in literature ${ }^{29,30}$. 
The weight loss data obtained from TGA for pure components and their blends are summarized in Table 2. It can be clearly seen that the blended film having CS/PVA ratio of $50 / 50$ shows the lowest amount of water content unlike blended films containing other ratios which show higher water content compared to pure chitosan film. A closer examination of Figure 3 reveals that all blends have only one onset (the degradation start temperature) suggesting that interaction may exist between the two components in each blend ${ }^{31}$ due to the formation of hydrogen bonding between the functional groups of the blended components ( $-\mathrm{OH}$ and $-\mathrm{NH}_{2}$ groups in chitosan and $-\mathrm{OH}$ groups in PVA).

Table 2. The weight loss $(\%)$ of the pure components and their blends at different temperatures

\begin{tabular}{cccccccc}
\hline Temperature, ${ }^{\circ} \mathrm{C}$ & Pure CS & $90 / 10$ & $80 / 20$ & $70 / 30$ & $60 / 40$ & $50 / 50$ & Pure PVA \\
\hline 100 & 5.7 & 6.1 & 6.9 & 6.3 & 6.2 & 3.9 & 3.1 \\
200 & 7.9 & 8.7 & 10.3 & 11.5 & 12.3 & 7.0 & 3.1 \\
300 & 30.4 & 32.7 & 35.6 & 39.0 & 38.7 & 31.0 & 7.1 \\
400 & 53.5 & 56.6 & 60.9 & 69.7 & 77.2 & 65.6 & 68.6 \\
500 & 58.3 & 61.3 & 67.1 & 76.8 & 86.3 & 74.9 & 92.8 \\
\hline
\end{tabular}

\section{Swelling behavior}

In this work as shown in Figure 4, all the blended films showed much higher degree of swelling comparing with the pure chitosan film, indicating their higher hydrophilicity. The degree of swelling for the blended films ranging from $1047 \%$ to $2117 \%$ while the degree of swelling for pure chitosan and pure PVA films were $89 \%$ and $674 \%$ respectively, which indicates that the swelling behavior is greatly influenced by the PVA content in the blend. This is because PVA is a water-soluble polymer and the blending of chitosan with PVA tends to increase the water uptake due to the increasing of hydrophilic groups $(-\mathrm{OH})$ in the blends. Also, the PVA chains are physically entangled with the chitosan chains leading to the formation of a hydrogel network ${ }^{32}$. It was reported that the blending of chitosan with the PVA $^{33,34}$ leads to an increase in the water uptake with increasing PVA content.

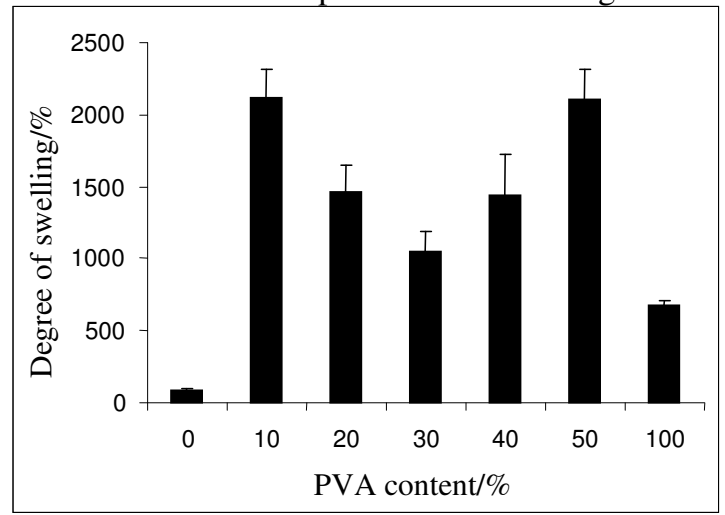

Figure 4. The swelling degree of chitosan, PVA and their blended films

\section{Conclusion}

Blended films of chitosan and PVA were prepared at various proportions. Investigation of the properties of the obtained blends displayed good miscibility between chitosan and PVA as revealed by the results of FTIR, FESEM and TGA due to the strong intermolecular hydrogen bonding existing between the amino groups of chitosan and the hydroxyl groups of PVA. Studying the swelling behavior showed much higher water uptake for all blended films compared to that of the pure chitosan film, indicating an enhancement in the hydrophilicity. 


\section{Acknowledgment}

The authors are grateful to University of Malaya for supporting this research project under the research grant, PS188/2008A.

\section{References}

1. Pillai O and Panchagnula R, Curr Opin Chem Biol., 2001, 5, 447.

2. Khor E and Lim L Y, Biomaterials, 2003, 24, 2339.

3. Yuan S and Wei T, J Bioact Compat Polym., 2004, 19, 467.

4. Crini G, Bioresour Technol., 2006, 97(9), 1061.

5. Araki C, Arai K and Hirase S, Bull Chem Soc Japan, 1967, 40, 959-962.

6. Stephen A. M, Phillips G O and Williams P A, Food Polysaccharides and Their Applications; Marcel Dekker Inc: New York, 1995, 187-204.

7. Park J S, Park J W and Ruckenstein E, Polym., 2001, 42, 4271.

8. Yamada K, Chen T, Kumar G, Vesnovsky O, Timmie L D and Payne G F, Biomacromol., 2000, 1, 253-258.

9. Mahdavinia G R, Pourjavadi A, Hosseinzadeh H and Zohuriaan M J, Eur Polym J., 2004, 40, 1399.

10. Huacai G, Wan P and Dengke L, Carbohydr Polym., 2006, 66, 372.

11. Sun T, Xu P X, Liu Q, Xue J A and Xie W M, Eur Polym J., 2003, 39, 189.

12. Yalpani M and Hall L D, Macromol., 1984, 17, 272.

13. Cheung M K, Wan K P Y and Yu P H, J Appl Polym Sci., 2002, 86, 1253-1258.

14. Suyatma N E, Copinet A, Tighzert L and Coma V, J Polym Env., 2004, 12(1), 1-6.

15. Smitha B, Dhanuja G and Sridhar S, Carbohydr Polym., 2006, 66, 463-472.

16. Ma L, Gao C Y, Mao Z W, Zhou J, Shen J C, Hu X Q and Han C M. Biomat., 2003, 24, 4833.

17. Muzzarelli R A A and Rochetti R, Carbohydr Polym., 1985, 5, 461-472.

18. Pawlak A and Mucha M, Thermochim Acta., 2003, 396, 153-166.

19. Nunthanid J, Puttipipatkhachorn S, Yamamoto and Peck G E, Drug Dev Ind Pharm., 2001, 27, 143-157.

20. Ritthidej G C, Phaechamud T and Koizumi T, Int J Pharm., 2002, 232(1-2), 11-22.

21. Xu Y X, Kim K M, Hanna M A and Nag D, Ind Crops Prod., 2005, 21, 185-192.

22. Ahmad A L and Ooi B S, J Membr Sci., 2005, 255, 67-77.

23. Guan Y L, Liu X F, Zhang Y P and Yao K D, J Appl Polym Sci., 1998, 67, 1965-1972.

24. Yin Y J, Yao K D, Cheng G X and Ma J B, Polym Int., 1999, 48, 429.

25. Peng F, Pan F, Sun, H, Lu L and Jiang Z, J Membr Sci., 2007, 300, 13-19.

26. Chen C H, Wang F Y, Mao C F, Liao W T and Hsieh C D, Int J Biol Macromol., 2008, 43, 37-42.

27. Britto D and Campana-Filho S P, Polym Degrad Stab., 2004, 84, 353.

28. Liu Y H, Shang Y J, Li W P, Wang Z and Deng K L, Chemical J Internet., 2002, 6, 27.

29. Shi R, Bi J, Zhang Z, Zhu A, Chen D, Zhou X, Zhang L and Tian W, Carbohydr Polym., 2008, 74. 763.

30. Zhou X-Y, Jia D- M, Cui Y-F and Xie D, J Reinf Plast Comp., 2009, 28, 2771.

31. Wu H, Wan Y, Cao X and Wu Q. Mater Lett., 2008, 62, 330.

32. Costa-Júnior E S, Barbosa-Stancioli E F, Mansur A A P, Vasconcelos W L and Mansur H S, Carbohydr Polym., 2009, 76, 472.

33. Wang T and Gunasekaran S, J Appl Polym Sci., 2006, 101, 3227.

34. Bahrami S B, Kordestani S S, Mirzadeh H and Mansoori P, Iran Polym J., 2005, 12, 139. 


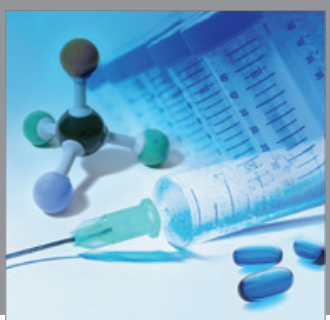

International Journal of

Medicinal Chemistry

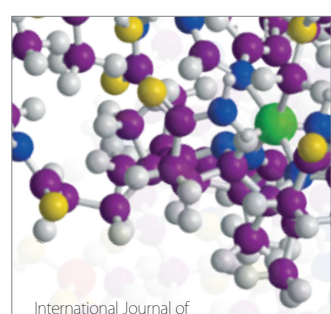

Carbohydrate Chemistry

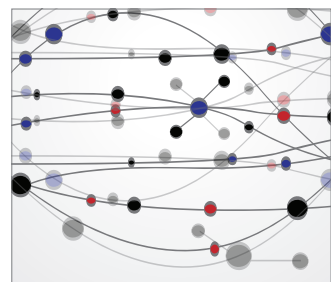

The Scientific World Journal
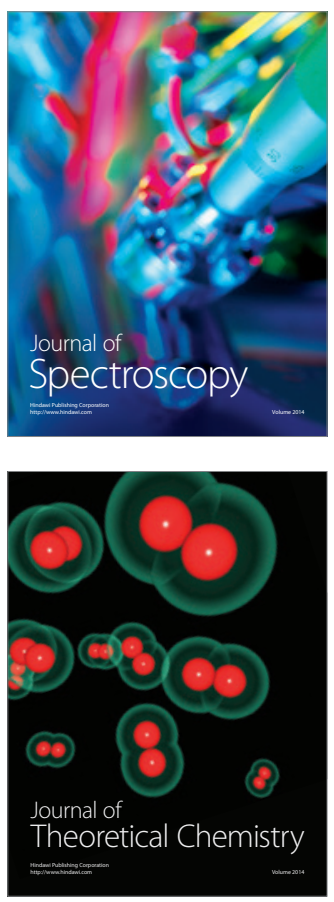
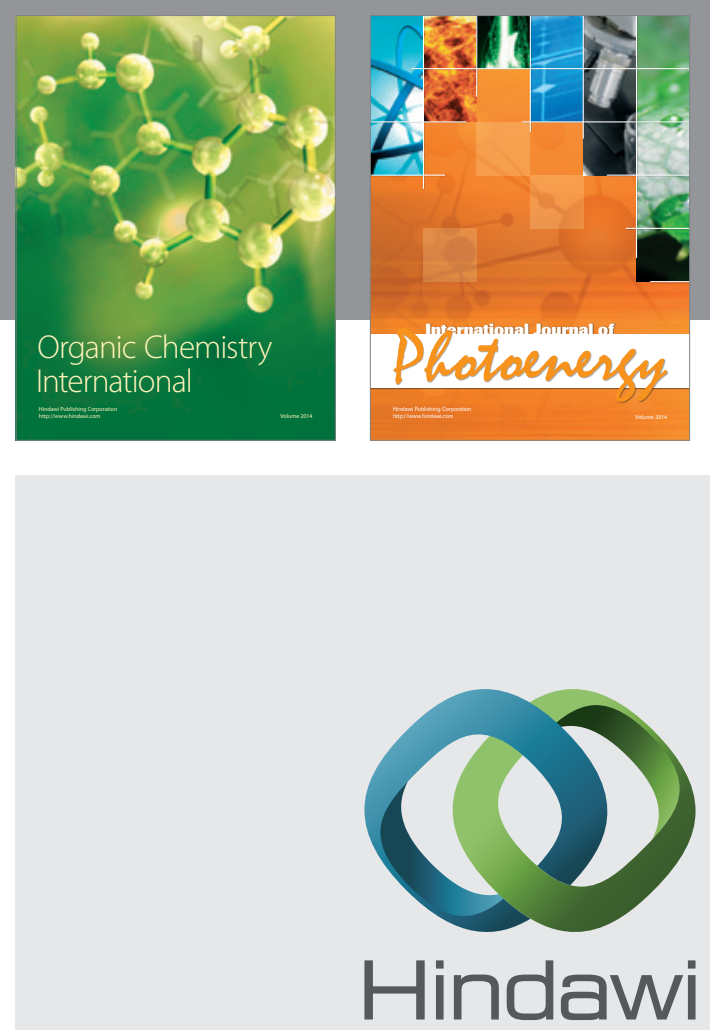

Submit your manuscripts at

http://www.hindawi.com
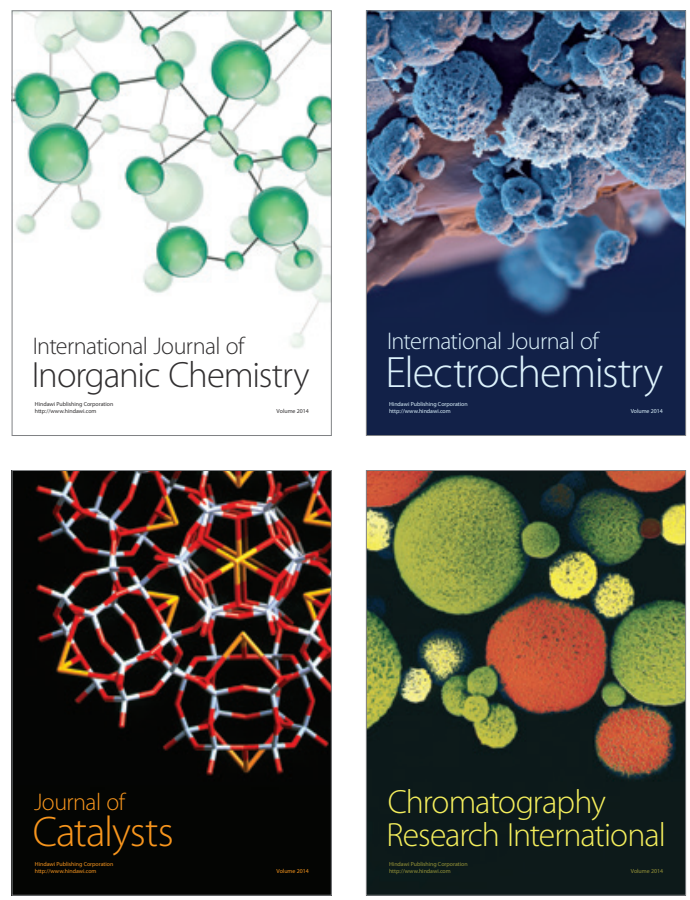
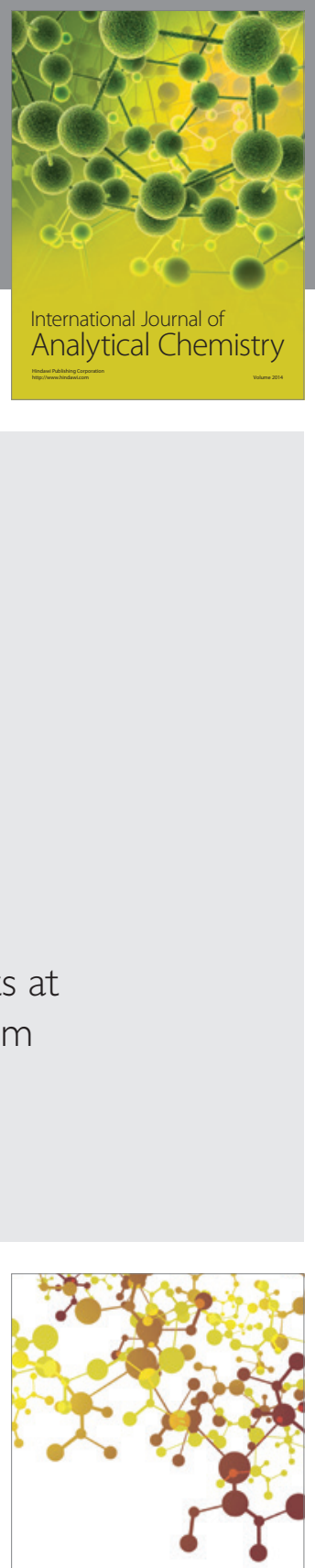

Journal of

Applied Chemistry
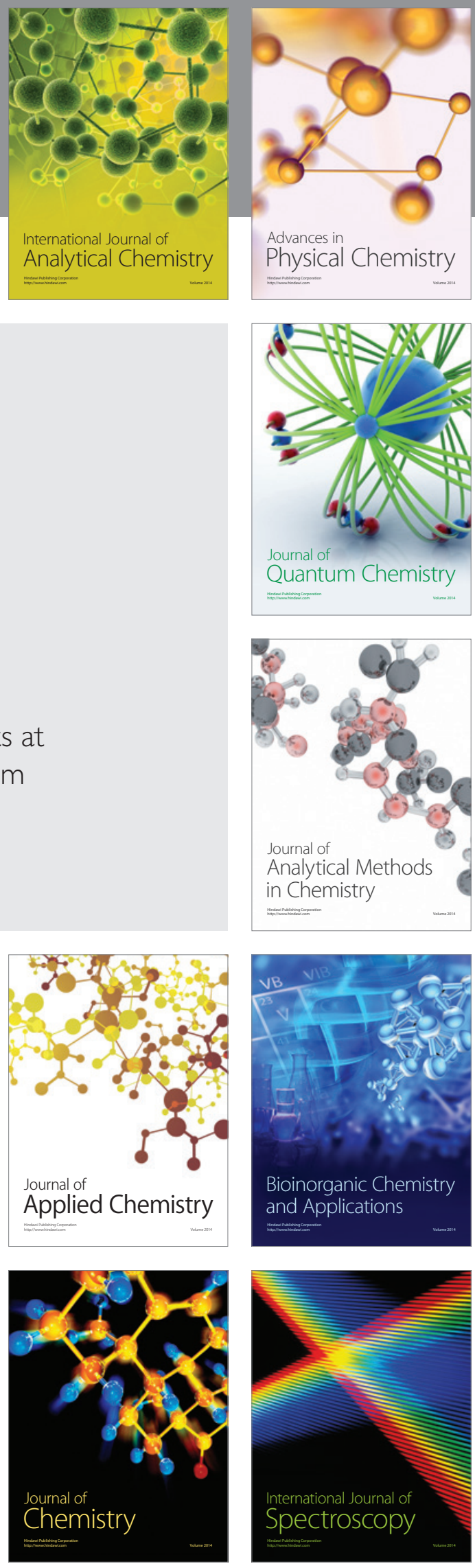